The Astrophysical Journal, 622:1033-1043, 2005 April 1

(C) 2005. The American Astronomical Society. All rights reserved. Printed in U.S.A.

\title{
PHOTON EMISSIVITY OF THE ELECTROSPHERE OF BARE STRANGE STARS
}

\author{
T. HARKo AND K. S. CHENG \\ Department of Physics, University of Hong Kong, Pokfulam Road, Hong Kong SAR, China; \\ harko@hkucc.hku.hk, hrspksc@hkucc.hku.hk \\ Received 2004 August 18; accepted 2004 December 11
}

\begin{abstract}
We consider the spectrum, emissivity, and flux of the electromagnetic radiation emitted by the thin electron layer (the electrosphere) at the surface of a bare strange star. In particular, we carefully consider the effect of the multiple and uncorrelated scattering on the radiation spectrum (the Landau-Pomeranchuk-Migdal effect), together with the effect of the strong electric field at the surface of the star. The presence of the electric field strongly influences the radiation spectrum emitted by the electrosphere. All the radiation properties of the electrons in the electrosphere essentially depend on the value of the electric potential at the quark star surface. The effect of the multiple scattering, which strongly suppresses radiation emission, is important only for the dense layer of the electrosphere situated near the star's surface and only for high values of the surface electric potential of the star. Hence a typical bremsstrahlung radiation spectrum, which could extend to very low frequencies, could be one of the main observational signatures even for low-temperature quark stars.
\end{abstract}

Subject headings: dense matter — elementary particles — radiation mechanisms: general

\section{INTRODUCTION}

The quark structure of nucleons, suggested by quantum chromodynamics (QCD), indicates the possibility of a hadron-quark phase transition at high densities and/or temperatures, as suggested by Itoh (1970), Bodmer (1971), Terazawa (1979), and Witten (1984). Theories of the strong interaction, such as, for example, the quark bag models, suppose that a breaking of the physical vacuum takes place inside hadrons. If the hypothesis of quark matter is true, then some neutron stars could actually be strange stars, built entirely of strange matter (Alcock et al. 1986; Haensel et al. 1986). For a general review of strange star properties, see Cheng et al. (1998a).

There are several proposed mechanisms for the formation of quark stars. Quark stars are expected to form during the collapse of the core of a massive star, after the supernova explosion, as a result of a first- or second-order phase transition, resulting in deconfined quark matter (Dai et al. 1995). The proto-neutron star core or the neutron star core is a favorable environment for the conversion of ordinary matter to strange quark matter (Cheng et al. 1998b). Another possibility is that some neutron stars in low-mass X-ray binaries can accrete sufficient mass to undergo a phase transition to become strange stars (Cheng \& Dai 1996). This mechanism has also been proposed by Cheng \& Dai (1998) as a source of radiation emission for cosmological $\gamma$-ray bursts. Quark stars can also be formed during the rapid spin-down of magnetars, astrophysical objects with extremely high magnetic fields (Harko et al. 2004).

Based on numerical integration of the general relativistic hydrostatic equilibrium equations, a complete description of the basic astrophysical properties (mass, radius, eccentricity, Keplerian frequency, etc.) of both static and rotating strange stars can be obtained (Witten 1984; Haensel et al. 1986; Gourgoulhon et al. 1999; Gondek-Rosinska et al. 2000; Zdunik 2000; Dey et al. 1998; Cheng \& Harko 2000; Harko \& Cheng 2002a). Rotational properties can discriminate between neutron and quark stars. Strange stars can reach much shorter periods than neutron stars, of the order of $0.5 \mathrm{~ms}$ (Cheng et al. 1998a). The $r$-mode instabilities in rapidly rotating strange stars lead to specific signatures in the evolution of pulsars with periods below $2.5 \mathrm{~ms}$. If strange matter is absolutely stable, pulsars would be expected to consist of quark matter. Some data on pulsar properties are consistent with this assumption (Madsen 2000a).

The structure of a realistic strange star is very complicated, but its basic properties can be described as follows (Alcock et al. 1986). Beta-equilibrated strange quark star matter consists of an approximately equal mixture of up $(u)$, down $(d)$, and strange $(s)$ quarks, with a slight deficit of the latter. The Fermi gas of $3 \mathrm{~A}$ quarks constitutes a single color-singlet baryon with baryon number $A$. This structure of the quarks leads to a net positive charge inside the star. Since stars in their lowest energy state are supposed to be charge neutral, electrons must balance the net positive quark charge in strange matter stars. The electrons, being bound to the quark matter by the electromagnetic interaction and not by the strong force, are able to move freely across the quark surface but clearly cannot move to infinity because of the electrostatic attraction of quarks. For hot stars the electron distribution could extend up to $\sim 10^{3} \mathrm{fm}$ above the quark surface (Cheng \& Harko 2003).

Photon emissivity is the basic parameter for determining macroscopic properties of stellar-type objects. Alcock et al. (1986) have shown that because of the very high plasma frequency $\omega_{p}$ near the strange matter edge, photon emissivity of strange matter is very low. Only photons produced just below the surface, in a quark layer of approximately $5 \mathrm{fm}$, and with momenta pointing outward can leave strange matter. For temperatures $T \ll E_{p} / \omega$, where $E_{p} \approx 23 \mathrm{MeV}$ is the characteristic transverse plasmon cutoff energy, the equilibrium photon emissivity of strange matter is negligibly small, as compared to the blackbody one. The spectrum of equilibrium photons is very hard, with $\hbar \omega>20 \mathrm{MeV}$.

The bremsstrahlung emissivity of quark matter has been estimated by Chmaj et al. (1991). They found that the surface radiation is about 4 orders of magnitude weaker than the equilibrium blackbody radiation, even though they both have the same temperature dependence. The problem of the soft photon emissivity of quark matter at the surface of strange stars has been reconsidered in Cheng \& Harko (2003). Taking into account the effect of the interference of amplitudes of nearby interactions in a dense 
medium (the Landau-Pomeranchuk-Migdal effect) and the absorption of the radiation in the external electron layer, the emissivity of the quark matter can be 6 orders of magnitude lower than the equilibrium blackbody radiation.

The Coulomb barrier at the quark surface of a hot strange star may also be a powerful source of $e^{+} e^{-}$pairs, which are created in the extremely strong electric field of the barrier. At surface temperatures of around $10^{11} \mathrm{~K}$, the luminosity of the outflowing plasma may be of the order of $\sim 10^{51}$ ergs s$^{-1}$ (Usov 1998a, 1998b, 2001). Moreover, as shown by Page \& Usov (2002), for about 1 day for normal quark matter and for up to $100 \mathrm{yr}$ for superconducting quark matter, the thermal luminosity from the star surface, because of both photon emission and $e^{+} e^{-}$pair production, may be orders of magnitude higher than the Eddington limit.

The existence of another major source of energy from a bare strange star, namely, the bremsstrahlung photon emission from the high-density electron layer at the surface of the star, has been pointed out by Jaikumar et al. $(2004,2005)$. The electron layer could be an important source of photons, which, because of the extremely low emissivity of quark-gluon plasma, could be the main observational signature of strange stars. The extreme-relativistic electrons of the electron layer undergo several types of collisions. The most important processes are the electron-electron elastic scattering, electron-photon scattering (Compton scattering), and electron-electron bremsstrahlung (together with its inverse). Of these three, the first two are assumed, on the average, to cause no net change of the gas, i.e., the electron and photon distributions remain unchanged. Hence the most important effect leading to the creation and subsequent loss of radiation in the electrosphere of the quark stars is the bremsstrahlung emission.

Since the electrons in the electrosphere of the bare strange stars have a high density, the effect of the dense medium plays an important role in the electromagnetic emission of the thin electron layer. The intensity of radiation from high-density systems can be significantly reduced by the so-called Landau-PomeranchukMigdal (LPM) effect (Landau \& Pomeranchuk 1953; Migdal 1956; Anthony et al. 1995; Klein 1999; Hansen et al. 2003; Cheng \& Harko 2003), which takes into account the effect of multiple collisions on the intensity of the emitted radiation. The influence of the LPM effect for the bremsstrahlung emissivity of the electrosphere has been discussed in Jaikumar et al. (2005), and it has been shown that the effect of the dense medium can reduce the emissivity by 2 orders of magnitude.

It is the purpose of the present paper to consider a systematic analysis of the LPM effect in the electrosphere of the bare quark stars. In particular, we would like to point out the effect of the electric field at the quark star surface on the electromagnetic radiation from the electrosphere. The presence of the electric field considerably reduces the magnitude of the LPM effect (e.g., the critical LPM frequency is reduced, because of the electric field, from $1 \mathrm{GeV}$ to a few $\mathrm{MeV}$ ).

The radiation properties of the electrosphere essentially depend on the value of the electric potential at the quark star surface. For high values of the electric potential (of the order of 16-20 MeV or higher), the electrosphere can be considered, from the point of view of the LPM effect, as a thick medium, and radiation is strongly suppressed in almost the entire volume of the electrosphere. On the other hand, for small values of the surface electrostatic potential of the quark star the electrosphere becomes thin, and the suppression effect can be ignored.

The present paper is organized as follows. In $\S 2$ we review the basic formalism and the main results describing the radiation spectrum of the individual electrons by taking into account the LPM effect. The emissivity and energy flux of the electro- sphere is obtained in $\S 3$. We discuss our results and conclude in $\S 4$. Throughout this paper we use the natural system of units, with $c=\hbar=k_{\mathrm{B}}=1$.

\section{PHOTON EMISSIVITY AND ELECTROMAGNETIC RADIATION ENERGY FLUX FROM THE ELECTROSPHERE OF QUARK STARS}

In this section we review some of the basic definitions and physical processes related to the electromagnetic radiation emission of the electrons in the dense electron layer at the surface of a quark star. In particular, we describe in some detail the mechanisms of electromagnetic radiation suppression due to the LPM effect in the case of electrons in a dense medium (Landau \& Pomeranchuk 1953; Migdal 1956), with and without the presence of an external electric field. This effect plays an essential role in the calculation of the radiation emission from the electrosphere of the quark star.

\subsection{Bremsstrahlung Emissivity and Energy Flux of the Electrosphere}

The emission of a photon in the electrosphere of a quark star is the result of the scattering of an electron from a state $\boldsymbol{p}_{1}$ to a state $\boldsymbol{p}_{1}^{\prime}$ by collision with another particle of momentum $\boldsymbol{p}_{2}$. In order to calculate the photon emissivity due to electron collisions in the electrosphere of quark stars, we consider the rate of collisions, $d N$, in which a soft photon of energy $\omega$ is emitted. According to the general principles of quantum electrodynamics, the rate of collisions is given by (Berestetskii et al. 1982)

$$
d N=\frac{1}{V} \int d W f_{1}\left(\epsilon_{1}\right) f_{2}\left(\epsilon_{2}\right)\left[1-f_{1}^{\prime}\left(\epsilon_{1}^{\prime}\right)\right]\left[1-f_{2}^{\prime}\left(\epsilon_{2}^{\prime}\right)\right],
$$

where

$$
\begin{aligned}
d W= & V(2 \pi)^{4} \delta^{(4)}\left(p_{1}+p_{2}-p_{1}^{\prime}-p_{2}^{\prime}-k\right)|M|^{2} \\
& \times \frac{d^{3} k}{(2 \pi)^{3} 2 \omega} \prod_{i=1,2,1^{\prime}, 2^{\prime}} \frac{d^{3} p_{i}}{(2 \pi)^{3} 2 \epsilon_{i}}
\end{aligned}
$$

is the scattering rate. We have denoted by $\left(\epsilon_{i}, \boldsymbol{p}_{i}\right)$ the $i$ th electron energy and momentum, where $\epsilon_{i}=\left(p_{i}^{2}+m^{2}\right)^{1 / 2}$, and by $(\omega, \boldsymbol{k})$ the photon energy and momentum. Here $f\left(\epsilon(p)-\mu_{e}\right)=$ $1 /\left(\exp \left\{\left[\epsilon(p)-\mu_{e}\right] / T\right\}+1\right)$ is the Fermi-Dirac distribution function for the $i$ th electron (Chiu 1968) and $M$ is the scattering amplitude. In order to obtain an explicit expression for the scattering rate, we adopt the method developed in Berestetskii et al. (1982), assuming that the photon emission process is quasi-classical. Consequently, for soft photon emission $d W$ can be written in a factorized form, $d W=d W_{0} d W_{\gamma}$, where $d W_{0}$ is the elastic electronelectron scattering rate and $d W_{\gamma}$ is the probability of emission of a photon of energy $\omega$ by any of the scattered electrons. The parameter $d W_{0}$ can be obtained from

$$
d W_{0}=V(2 \pi)^{4} \delta^{(4)}\left(p_{1}+p_{2}-p_{1^{\prime}}-p_{2^{\prime}}\right)\left|M_{0}\right|^{2} \prod_{i=1,2,1^{\prime}, 2^{\prime}} \frac{d^{3} p_{i}}{(2 \pi)^{3} 2 \epsilon_{i}},
$$

where $M_{0}$ is the electron-electron scattering amplitude. The reaction rate can be expressed in a more convenient form in terms of the cross section $\sigma$ of the reaction. 
In terms of the cross section $\sigma$ of the emission process, the rate of collisions in the electron plasma in which a photon of energy $\omega \ll \epsilon$ is emitted can be obtained as (Chiu 1968)

$$
\begin{aligned}
d N= & g_{1} g_{2} f_{1}\left(\boldsymbol{p}_{1}\right) f_{2}\left(\boldsymbol{p}_{2}\right) \sigma(\theta, \phi)\left|\boldsymbol{v}_{1}-\boldsymbol{v}_{2}\right|\left[1-f_{1}\left(\boldsymbol{p}_{1}^{\prime}\right)\right] \\
& \times\left[1-f_{2}\left(\boldsymbol{p}_{2}^{\prime}\right)\right] \prod_{i=1,2,1^{\prime}, 2^{\prime}} d^{3} \boldsymbol{p}_{i} d \Omega(\theta, \phi),
\end{aligned}
$$

where $d \Omega(\theta, \phi)$ is the solid angle element in the direction $(\theta, \phi), g_{1}$ and $g_{2}$ are the statistical weights, and $\boldsymbol{v}_{1}$ and $\boldsymbol{v}_{2}$ are the velocities of the interacting particles. The invariant relative velocity $v_{r}=$ $\left|\boldsymbol{v}_{1}-\boldsymbol{v}_{2}\right|$ is defined by $v_{r}=\left\{\left[\left(p_{1} \cdot p_{2}\right)^{2}-m_{e}^{4}\right] /\left(p_{1} \cdot p_{2}\right)^{2}\right\}^{1 / 2}$, where $p_{1}$ and $p_{2}$ are the energy-momentum four-vectors of the two electrons (Landau \& Lifshitz 1975). The metric has been chosen so that the product of two four-vectors $a^{\mu}$ and $b^{\mu}$ is defined by $a \cdot b=a_{0} b_{0}-\boldsymbol{a} \cdot \boldsymbol{b}$. The energy $\epsilon$ of the electron in the center of mass system is related to the four-momenta in an arbitrary frame by $\epsilon=\left[\left(p_{1} \cdot p_{2}+1\right) / 2\right]^{1 / 2}$ (Landau \& Lifshitz 1975). Therefore, in order to calculate the reaction rate for the photon emission process we need to know the cross section of this process.

Let $d \sigma_{0}$ be the cross section for a given process of scattering of charged particles, which may be accompanied by the emission of a certain number of photons. For example, $d \sigma_{0}$ could refer to the scattering of an electron by another electron, with the possible emission of hard photons. Together with this process, one could consider another process that differs from it only in that one extra photon is emitted. In this case the total cross section $d \sigma$ can be represented as a product of two independent factors, the cross section $d \sigma_{0}$ and the probability $d W_{\gamma}$ of emission of a single photon in the collision (Berestetskii et al. 1982). The emission of a soft photon is a quasi-classical process. The probability of emission is the same as the classically calculated number of quanta emitted in the collision, that is, the same as the classical intensity (total energy) of emission $d I$, divided by the frequency of the radiation $\omega$ (Berestetskii et al. 1982).

The probability $d W_{\gamma}$ of emitting a photon of energy $\omega$ by any of the scattered electrons is $d W_{\gamma}=d I / \omega$. Therefore, the total cross section for the emission of soft photons is given by (Berestetskii et al. 1982)

$$
d \sigma=d \sigma_{0} \frac{d I}{\omega}
$$

To calculate the total cross section for a photon emission we need to know $d \sigma_{0}$, describing the elastic scattering of electrons. We assume that $d \sigma_{0}$ is equal to the electron-electron elastic scattering cross section $d \sigma_{\mathrm{el}}$, given, in the center of mass system, in which the magnitudes of the momentum $\boldsymbol{p}$ and energy $\epsilon$ of the electrons are unchanged in the scattering, by (Berestetskii et al. 1982)

$$
\begin{aligned}
d \sigma_{\mathrm{el}}= & r_{e}^{2} \frac{m_{e}^{2}\left(\epsilon^{2}+\boldsymbol{p}^{2}\right)^{2}}{4 \boldsymbol{p}^{4} \epsilon^{2}}\left[\frac{4}{\sin ^{4} \theta}-\frac{3}{\sin ^{2} \theta}\right. \\
& \left.+\left(\frac{\boldsymbol{p}^{2}}{\epsilon^{2}+\boldsymbol{p}^{2}}\right)^{2}\left(1+\frac{4}{\sin ^{2} \theta}\right)\right] d \Omega .
\end{aligned}
$$

By assuming that the energies of the electrons in the electrosphere are ultrarelativistic, $\boldsymbol{p}^{2}=\epsilon^{2}$, we obtain from equation (5) the limiting form $d \sigma_{\mathrm{el}}=\left(r_{e}^{2} m_{e}^{2} / \epsilon^{2}\right)\left(3+\cos ^{2} \theta\right)^{2} d \Omega / 4 \sin ^{4} \theta$. There- fore, the total cross section for the emission of a photon can be written as

$$
\sigma=\int d \sigma_{\mathrm{el}}\left\langle\frac{d I}{d \omega}\right\rangle \frac{d \omega}{\omega},
$$

where the factor $\langle d I / d \omega\rangle$ describes the intensity of the radiation emitted by the electron in multiple collisions.

We assume that the electrons form a degenerate Fermi gas, with the particle number density $n$ given by (Chiu 1968)

$$
\begin{aligned}
n & =\frac{g}{2 \pi^{2}} \int_{0}^{\infty} f\left(\epsilon(p)-\mu_{e}\right) p^{2} d p \\
& =\frac{g}{2 \pi^{2}} \int_{0}^{\infty} \frac{p^{2} d p}{\exp \left\{\left[\epsilon(p)-\mu_{e}\right] / T\right\}+1} .
\end{aligned}
$$

The emissivity per unit volume of the electrosphere can be calculated by using the definition

$$
\varepsilon_{\gamma}^{(e e)}(z, T)=\frac{d E_{\gamma}^{e e}}{d t d V}=\int \omega d N
$$

The energy flux from the electrosphere of a strange star, coming out from a thin surface layer of thickness $d z$, is $F_{\gamma}^{(e e)}=\varepsilon_{\gamma}^{(e e)} d z / \pi$. Taking into account the contribution of all layers, we find

$$
F_{\gamma}^{(e e)}(T)=\frac{1}{\pi} \int_{0}^{\infty} \varepsilon_{\gamma}^{(e e)}(z, T) d z .
$$

\subsection{Radiation Intensity of Charged Particles in Dense Media}

As a first step in obtaining the photon emissivity of the electrosphere of the bare strange stars, we have to obtain the classical radiation intensity distribution $\langle d I / d \omega\rangle$ emitted by electrons moving in a dense medium in which many interparticle collisions occur. Since in the electrosphere a very strong electric field is also present, the influence of this field on the radiation process must also be taken into account.

In the early 1950s Landau \& Pomeranchuk (1953) and Migdal (1956) predicted that the radiation from highly relativistic particles in dense media is suppressed because of interference between amplitudes of nearby interactions (for detailed presentations of the LPM effect, see the monograph by Akhiezer \& Shul'ga [1996] and the review article by Klein [1999]). The suppression effect is of a quantum mechanic nature and has its roots in the uncertainty principle. The kinematics of the bremsstrahlung requires that the longitudinal momentum transfer between the interacting particles must be small; the uncertainty principle then requires that the interaction must occur over a large longitudinal distance scale (formation zone). If the electron Coulomb scatters while traversing this zone, the bremsstrahlung amplitude from before and after scattering can interfere, thus reducing the amplitude for bremsstrahlung photon emission (Akhiezer \& Shul'ga 1996 and references therein). The results of quantitative measurements of the bremsstrahlung suppression due to the LPM effect for electrons with energies of 8 and $25 \mathrm{GeV}$ traversing thin gold and carbon targets have been presented in Anthony et al. (1995). The suppression of bremsstrahlung predicted by the LPM theory is correct to within $5 \%$.

The main parameters defining radiation processes in a medium are the coherence length $l_{c}$, the mean free path of a fast particle in matter $l_{e}$, the radiation length $X_{0}$, and the thickness of the target $L$ (Akhiezer \& Shul'ga 1996 and references therein). The coherence length is the distance along the particle momentum where 
interference effects during the radiation process are significant. If the energy $\omega$ of the bremsstrahlung photon produced by an ultrarelativistic particle of energy $E$ satisfies the condition $\omega \ll \epsilon$, then the average angle $\theta$ between the incident particle and the photon is small, $\theta_{c} \approx m c^{2} / \epsilon$. The average angle between the scattered particles is smaller still. Neglecting these angles, the longitudinal momentum transfer between the interacting particles is $p_{\|} \simeq \omega / 2 \gamma^{2} c$, where $\gamma=\epsilon / m c^{2}$. The uncertainty principle then requires that the spatial position of the bremsstrahlung process has a longitudinal uncertainty of $l_{c}=\hbar / p_{\|} \approx 2 \hbar c \gamma^{2} / \omega$. The coherence length rapidly grows with an increase of the particle energy, and at high energies can be macroscopic. In alternate language, the particle and photon slowly split apart over the distance $l_{c}$. In a sufficiently dense medium the mean free path of the incident particle is much smaller than $l_{c}$, so a particle will interact while traversing the region $l_{c}$. Bremsstrahlung is suppressed when the mean-square multiple scattering angle over the distance $l_{c}$,

$$
\theta_{s}^{2}=\left(\frac{\epsilon_{s}}{\epsilon}\right)^{2} \frac{l_{c}}{X_{0}}
$$

is greater than or equal to $\theta_{k}^{2}$ (Akhiezer $\&$ Shul'ga 1996; Klein 1999 and references therein). Here

$$
X_{0}=\left[4 n \alpha r_{e}^{2} Z^{2} \ln \left(184 Z^{-1 / 3}\right)\right]^{-1}
$$

is the radiation length, and

$$
\epsilon_{s}=m c^{2} \sqrt{\frac{4 \pi}{\alpha}} .
$$

In equation (12), $r_{e}=e^{2} / m c^{2}$ is the classical radius of the particle (the electron). The effect of multiple suppression on the total radiation emission of the charged particle, $d I / d \omega$, can be obtained, for $\omega \ll \epsilon_{s}^{2} c / \epsilon^{2} X_{0}$, in the form (Klein 1999 and references therein)

$$
\frac{d I}{d \omega}=\sqrt{\frac{2 \pi}{3}} \frac{Z^{2} e^{2} m^{2} c^{3}}{\epsilon_{s} \epsilon} \sqrt{\frac{\omega X_{0}}{c}} .
$$

Thus, multiple scattering results in a decrease of the coherence length, leading, in turn, to radiation suppression. The effect appears in the case when the mean-square angle of the multiple scattering within the coherence length $\left\langle\theta^{2}\right\rangle$ exceeds the characteristic squared angle of the relativistic electron radiation $\theta^{2} \sim$ $1 / \gamma^{2}$ (Landau \& Pomeranchuk 1953; Klein 1999). The suppression happens when the frequency of the radiation satisfies the condition

$$
\omega<\omega_{\mathrm{LPM}}=\frac{\epsilon_{s}^{2} \epsilon}{m^{4} c^{7} X_{0}} .
$$

The electromagnetic radiation of charged particles in external fields plays an essential role in astrophysical processes, the most important cases being the synchrotron radiation in a constant magnetic field or the unified synchro-curvature radiation of electrons in constant electric and magnetic fields (Harko \& Cheng $2002 b$ ). However, the radiation of electrons in pure electric fields $\boldsymbol{E}$ has been less studied.

The intensity of the radiation of charged particles in dense media in the presence of an external electric field and with the LPM effects included was obtained by Baryshevskii \& Tikhomirov (1986) and later on by Ha (1997). In the present paper we closely follow the presentation and the derivation of Baryshevskii \& Tikhomirov (1986). Because of the presence of the electric field, there is a decrease of the influence of multiple scattering on the process of radiation.

This important result can be understood qualitatively as follows (Baryshevskii \& Tikhomirov 1986). In a dense medium the condition for the suppression of the radiation due to multiple scattering is $\left\langle\theta^{2}\left(l_{c}\right)\right\rangle \geq \theta_{\gamma}^{2}$, where $\left\langle\theta^{2}\right\rangle$ is given by equation (11) and $l_{c} \approx 2 \gamma^{2} / \omega \approx 2 \epsilon^{2} / \mathrm{m}^{2} \omega$ is the coherence length. In obtaining this relation we have assumed that the scattering angles are randomly oriented in the plane perpendicular to the direction of motion of the particle.

For sufficiently high electron energies we can approximate $\omega \approx$ $\epsilon$, thus obtaining $l_{c} \approx 2 \epsilon / m^{2}$ and $\left\langle\theta^{2}\left(l_{c}\right)\right\rangle \propto \epsilon^{-1}$. Since $\theta_{\gamma}^{2} \propto \epsilon^{-2}$, at sufficiently high energies the condition $\left\langle\theta^{2}\left(l_{c}\right)\right\rangle \geq \theta_{\gamma}^{2}$ will always be satisfied.

However, this situation radically changes in the case of a particle in an external electric field. Radiation in an electric field of intensity $E$ is characterized by the parameter

$$
\zeta=\frac{e E \epsilon}{m^{3}}=\frac{\gamma E}{E_{\mathrm{cr}}},
$$

where $E_{\mathrm{cr}}=m^{2} / e$ is the critical electric field (Berestetskii et al. 1982). For electrons, the value of $E_{\mathrm{cr}}$ is $1.3 \times 10^{16} \mathrm{~V} \mathrm{~cm}^{-1}$. If $\zeta \geq 1$, then the energy of the radiated gamma rays is $\omega \sim$ $\zeta \epsilon /(1+\zeta) \sim \epsilon$, and the radiation process is essentially quantum in nature.

For $\zeta \gg 1$ the characteristic angle of the radiation $\theta_{\gamma}$ is modified to $\theta_{E} \propto \theta_{\gamma} \zeta^{1 / 3}$, and the coherence length becomes $l_{E} \propto$ $\omega / m \zeta^{2 / 3}$ (Baryshevskii \& Tikhomirov 1986). Multiple scattering will greatly change the pattern of formation of radiation for $\left\langle\theta^{2}\left(l_{E}\right)\right\rangle \geq \theta_{E}^{2}$. Since $\zeta \sim \epsilon$, we have $\left\langle\theta^{2}\left(l_{E}\right)\right\rangle \propto \epsilon^{-5 / 3}$ and $\theta_{E}^{2} \propto$ $\epsilon^{-4 / 3}$; i.e., in contrast to the case of a dense medium without an external field, the left-hand side of the inequality falls off with the increase of the energy $\epsilon$ faster than the right side. The suppression of the LPM effect in the presence of the electric field can be interpreted as an effective shortening of the coherence length, because in a strong electric field the $1 / \gamma$ emission zone shortens: the emitting particle is deviated out of the emission cone by the field. Therefore, with the increase of $\epsilon$ and $\omega$ we should expect only a decrease of the influence of the multiple scattering on the process of radiation (Baryshevskii \& Tikhomirov 1986; Ha 1997).

By using the distribution function for the particles in an electric field, the average probability over all possible trajectories can be written as a functional integral over a Wiener measure. The calculations have been done in Baryshevskii \& Tikhomirov (1986) and Ha (1997), and the resulting expression for the probability of radiation of a photon by a charged particle moving in a uniform electric field and undergoing multiple scattering is

$$
\begin{aligned}
\left\langle\frac{d I}{d \omega}\right\rangle= & -\frac{e^{2} m^{2}}{\sqrt{\pi} \epsilon^{2}}\left[F_{1}(r, \eta, x)+\left(\frac{2}{x}+\frac{\omega}{\epsilon} \zeta \sqrt{x}\right) f_{3}(r, \eta, x)\right. \\
& \left.+\frac{\epsilon^{2}+\epsilon^{\prime 2}}{3 \omega \epsilon^{2} L\left(\sigma_{x}\right)} F_{2}(r, \eta, x)\right]
\end{aligned}
$$

where $\sigma_{i}$ for $i=x$ and $y$ represent the mean squares of the angles of deviation from the direction of motion of the particle in the average potential acquired per unit length, $\sigma_{i}=d\left\langle\theta_{s i}^{2}\right\rangle / d z$ for $i=x$ and $y$, 
$r=\left(\sigma_{y} / \sigma_{x}\right)^{1 / 2}, \quad \epsilon^{\prime}=\epsilon-\omega, \quad \eta=m^{2} \omega / \epsilon \epsilon^{\prime}\left(2 \omega \epsilon \sigma_{x} / \epsilon^{\prime}\right)^{1 / 2}, \quad x=$ $\left(m^{3} \omega / e E \epsilon \epsilon^{\prime}\right)^{2 / 3}, L\left(\sigma_{x}\right)=\pi m^{2} / e^{2} \epsilon^{2} \sigma_{x}$, and

$$
\begin{aligned}
F_{1}(r, \eta, x)= & \frac{2}{\sqrt{\pi}} \int_{0}^{\infty} e^{-\eta t}\left\{\frac{\sin \eta t}{2 t}\right. \\
& -f(r, t) \exp [\varphi(x, \eta, t)] \sin [\eta t+\varphi(x, \eta, t)]\} d t,
\end{aligned}
$$

$$
\begin{aligned}
F_{2}(r, \eta, x)= & 3 \sqrt{2} \eta \int_{0}^{\infty}[\tanh (t)+r \tanh (r t)] f(r, t) \\
& \times \exp [-\eta t+\varphi(x, \eta, t)] \cos \left[\eta t+\varphi(x, \eta, t)-\frac{\pi}{4}\right] d t,
\end{aligned}
$$

$$
\begin{aligned}
F_{3}(r, \eta, x)= & -\frac{4 \eta^{2}}{\sqrt{\pi} x^{2}} \int_{0}^{\infty} \tanh ^{2}(t) f(r, t) \\
& \times \exp [-\eta t+\varphi(x, \eta, t)] \cos \left[\eta t+\varphi(x, \eta, t)-\frac{\pi}{4}\right] d t,
\end{aligned}
$$

$$
\begin{aligned}
f(r, t) & =\sqrt{\frac{r}{\sinh (2 t) \sinh (2 r t)},} \\
\varphi(x, \eta, t) & =\frac{2 \eta^{3}}{x^{3}}[\tanh (t)-t] .
\end{aligned}
$$

These equations give a complete description of the radiation pattern emitted by a charged particle moving in a dense medium in the presence of an electric field.

In the limit of the dominance of the electric field and symmetric multiple scattering we have $x<1$ and $x \ll \eta$, and equation (17) goes into the expression for the intensity of the radiation in a constant electromagnetic field, also taking into account quantum effects, by (Berestetskii et al. 1982)

$$
\frac{d I}{d \omega}=-\frac{e^{2} m^{2} \omega}{\sqrt{\pi} \epsilon^{2}}\left[\int_{x}^{\infty} \operatorname{Ai}(y) d y+\left(\frac{2}{x}+\frac{\omega}{\epsilon} \zeta \sqrt{x}\right) \operatorname{Ai}^{\prime}(x)\right],
$$

where $x=\left(\omega / \epsilon^{\prime} \zeta\right)^{2 / 3}, \epsilon^{\prime}=\epsilon-\omega$, and $\operatorname{Ai}(y)=\int_{0}^{\infty} \cos (y t+$ $\left.t^{3} / 3\right) d t / \pi^{1 / 2}$ is the Airy function. In the classical limit, $\omega \ll$ $\epsilon^{\prime} \approx \epsilon$, and $x \approx\left(\omega / \omega_{0}\right)^{2 / 3}(m / \epsilon)^{2}$, where $\omega_{0}=e \zeta / \epsilon$. In this case the second term in the parentheses in equation (22) is small, and equation (22) goes to the classical formula for the electromagnetic radiation of an electron in an external electromagnetic field (Landau \& Lifshitz 1975).

\section{SINGLE-ELECTRON RADIATION, EMISSIVITY, AND ENERGY FLUX FROM THE ELECTROSPHERE OF QUARK STARS}

In this section we consider an example of how the formalism described in $\S 2$ can be used to describe the radiation properties of the electrosphere of the bare strange stars.

\subsection{Structure of the Electrosphere of Quark Stars}

For the electron distribution in the electrosphere of the bare strange stars we use the model presented in Cheng \& Harko (2003), which also takes into account the finite-temperature effects and can be solved exactly, with all physical quantities of interest (chemical potential, electric field, etc.) expressed in an exact analytical form. The chemical equilibrium implies that the electron chemical potential $\mu_{\infty}=-V+\mu_{e}$ is constant, where $V$ is the electrostatic potential per unit charge and $\mu_{e}$ is the electron's chemical potential. Since far outside the star both $V$ and $\mu_{e}$ tend toward zero, it follows that $\mu_{\infty}=0$ and $\mu_{e}=V$ (Alcock et al. 1986).

The Poisson equation for the electrostatic potential $V(z, T)$ generated by the finite-temperature electron distribution reads (Alcock et al. 1986; Kettner et al. 1995)

$$
\begin{gathered}
\frac{d^{2} V}{d z^{2}}=\frac{4 \alpha}{3 \pi}\left[\left(V^{3}-V_{q}^{3}\right)+\pi^{2}\left(V-V_{q}\right) T^{2}\right], \quad z \leq 0, \\
\frac{d^{2} V}{d z^{2}}=\frac{4 \alpha}{3 \pi}\left(V^{3}+\pi^{2} T^{2} V\right), \quad z \geq 0,
\end{gathered}
$$

where $T$ is the temperature of the electron layer, which can be taken as a constant, since we assume that the electrons are in thermodynamic equilibrium with the constant-temperature quark matter. In equations (23) and (24), $z$ is the spatial coordinate measuring the height above the quark surface, $\alpha$ is the fine-structure constant, and $V_{q} / 3 \pi^{2}$ is the quark charge density inside the quark matter. The boundary conditions for equations (23) and (24) are $V \rightarrow V_{q}$ as $z \rightarrow-\infty$ and $V \rightarrow 0$ for $z \rightarrow \infty$. In the case of the zero-temperature electron distribution at the boundary $z=0$ we have the condition $V(0)=(3 / 4) V_{q}$ (Alcock et al. 1986).

The structure of the electrosphere and the corresponding radiation processes essentially depends on the value of $V_{q}$, the electric charge density inside the quark star. When the temperature of the quark star core drops below $10^{9} \mathrm{~K}$, the strange matter becomes superfluid. At this temperature quarks can form colored Cooper pairs near the Fermi surface and become superconducting. From the Bardeen-Cooper-Schrieffer (BCS) theory it follows that the critical temperature $T_{c}$ at which the transition to the superconducting state takes place is $T_{c}=\Delta / 1.76$, where $\Delta$ is the pairing gap energy (Blaschke et al. 2000). An early estimation of $\Delta$ gave $\Delta \sim 0.1-1 \mathrm{MeV}$ (Bailin \& Love 1984), but some recent studies considering instanton-induced interactions between quarks estimated $\Delta \sim 100 \mathrm{MeV}$ (Alford et al. 1998).

Strange quark matter in the color-flavor locked (CFL) phase of QCD, which occurs for $\Delta \sim 100 \mathrm{MeV}$, could be rigorously electrically neutral, despite the unequal quark masses, even in the presence of the electron chemical potential (Alford et al. 1998). Hence, for the CFL state of quark matter, $V_{q}=0$, and no electrons are present inside the quark star.

However, Page \& Usov (2002) pointed out that for sufficiently large $m_{s}$, the low-density regime is rather expected to be in the "2-color-flavor Superconductor" phase, in which only $u$ and $d$ quarks of two colors are paired in a single condensate, while the ones of the third color and $s$ quarks of all three colors are unpaired. In this phase, some electrons are still present. In other words, electrons may be absent in the core of strange stars but present at least near the surface, where the density is lowest. Nevertheless, the presence of the CFL effect can reduce the electron density at the surface, and hence it can also significantly reduce the electromagnetic emissivity of the electrons in the surface layer. Therefore, in order to describe the radiation properties of the electrosphere, we assume that $V_{q} \neq 0$.

The general solution of equation (24) is given by (Cheng \& Harko 2003)

$$
V(z, T)=\frac{2 \sqrt{2} \pi T \exp \left[2 \sqrt{\alpha \pi / 3} T\left(z+z_{0}\right)\right]}{\exp \left[4 \sqrt{\alpha \pi / 3} T\left(z+z_{0}\right)\right]-1},
$$

where $z_{0}$ is a constant of integration. Its value can be obtained from the condition of the continuity of the potential across the 


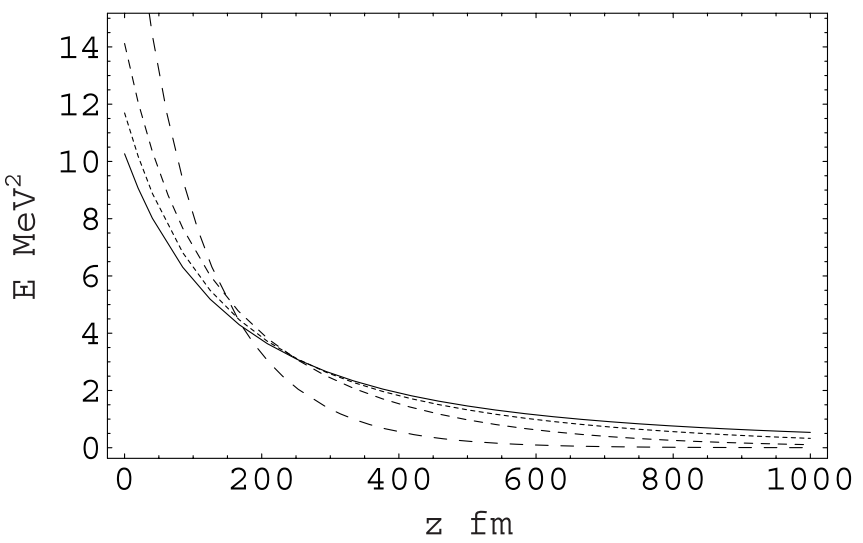

FIG. 1.-Electric field $E$ (in units of $\mathrm{MeV}^{2}$ ) as a function of distance $z$ (fm) for different values of the temperature: $T=1$ (solid curve), 3 (dotted curve), 5 (dashed curve), and $10 \mathrm{MeV}$ (long-dashed curve). In all cases $V_{I}(0, T)=16 \mathrm{MeV}$.

star's surface, requiring $V_{I}(0, T)=V(0, T)$, where $V_{I}(z, T)$ is the value of the electrostatic potential in the region $z \leq 0$, described by equation (23). Therefore,

$$
z_{0}=\frac{1}{2} \sqrt{\frac{3}{\alpha \pi}} \frac{1}{T} \ln \left[\frac{\sqrt{2} \pi T}{V_{I}(0, T)}\left(1+\sqrt{1+\frac{V_{I}^{2}(0, T)}{2 \pi^{2} T^{2}}}\right)\right] .
$$

The number density distribution $n_{e}$ of the electrons at the quark star surface can be obtained from $n_{e}(z, T)=V^{3} / 3 \pi^{2}+V T^{2} / 3$ (Kettner et al. 1995; Cheng \& Harko 2003).

In the limit of zero temperature, $T \rightarrow 0$, we obtain $V(z)=$ $a_{0} /(z+b)$, where $a_{0}=(3 \pi / 2 \alpha)^{1 / 2}$ and $b$ is an integration constant. The value of $b$ can be determined from the boundary condition $V(0)=(3 / 4) V_{q}$, which gives $b=4 a_{0} / 3 V_{q}$. Therefore, in this case for the electron particle number distribution, extending several thousands of fermis above the quark matter surface, we find the expression $n_{e}(z)=\left(1 / 3 \pi^{2}\right) a_{0}^{3} /(z+b)^{3}$. In the absence of a crust of the quark star, the electron layer can extend to several thousands of fermis outside the star's surface.

The variation of the strength of the electric field $E$ outside the quark star surface is given by

$$
E(z, T)=\sqrt{\frac{2 \alpha}{3 \pi}} V \sqrt{V^{2}+\pi^{2} T^{2}}
$$

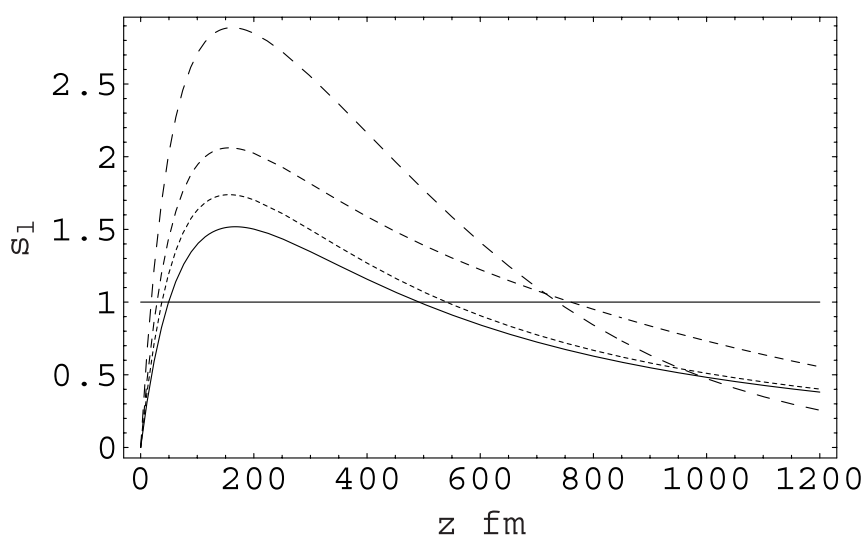

The electric field is presented as a function of the distance from the quark star surface and for different values of the temperature in Figure 1.

\subsection{Single-Electron Radiation and Emissivity of the Electrosphere of Bare Strange Stars}

In order to study the electromagnetic radiation emission from the electrosphere of the quark stars and the physical processes determining it, it is necessary to find whether, with respect to the LPM effect, the electrosphere is a thin or thick medium. In extremely thin media, neither dielectric effects nor multiple scattering produce enough of a phase shift to cause suppression of radiation. Suppression due to multiple scattering disappears when the total scattering angle in the target is less than $1 / \gamma$. This happens when the thickness $l$ of the dense medium is smaller than a critical thickness $l_{c}(z, T)$ (Klein 1999 and references therein):

$$
\begin{aligned}
l<l_{\text {crit }}(z, T) & =\left(\frac{m_{e}}{\epsilon_{s}}\right)^{2} X_{0} \\
& =\frac{1}{4 \pi}\left[4 n(z, T) r_{e}^{2} Z^{2} \ln \left(184 Z^{-1 / 3}\right)\right]^{-1}
\end{aligned}
$$

To describe the general properties of the electrosphere of the quark stars with respect to the effect of the multiple scattering on electromagnetic radiation, we introduce a parameter called the suppression factor and defined as

$$
s_{l}(z, T) \equiv \frac{z}{l_{\text {crit }}}=4 \pi z\left[4 n(z, T) r_{e}^{2} Z^{2} \ln \left(184 Z^{-1 / 3}\right)\right]
$$

The LPM effect is important for regions for which $s_{l}(z, T) \geq 1$; for regions in the electrosphere with $s_{l}(z, T)<1$, the LPM effect can be safely ignored. The variation of the suppression factor $s_{l}$ as a function of $z$ for two sets of values of the surface electric potential of the quark star and for different values of the temperature is presented in Figure 2.

As one can see from Figure 2, the effect of the multiple scattering is extremely important in the dense layer situated near the quark star surface. For the electrons situated far away from the surface, the suppression due to multiple scattering can be ignored.

In the absence of an external field, electromagnetic radiation emission of electrons is strongly suppressed when the mean-square multiple scattering angle over the distance

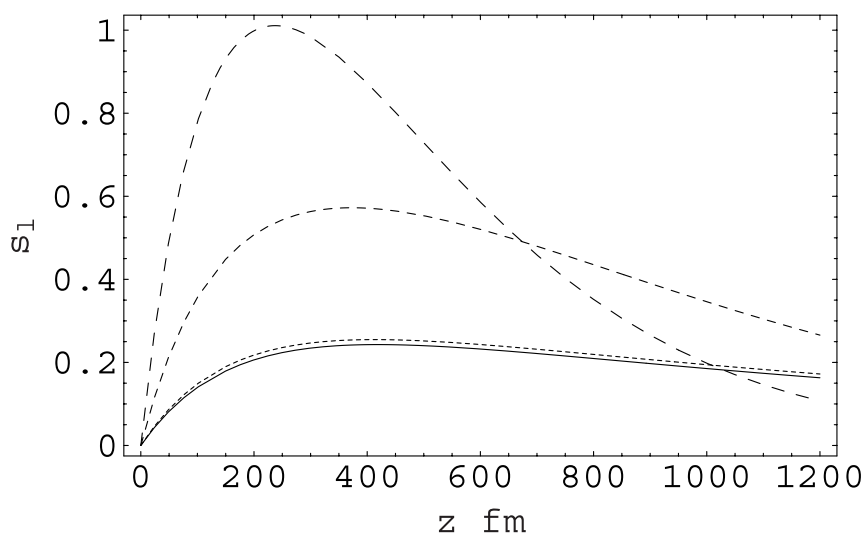

FIG. 2.- Variation of the suppression factor $s_{l}$ for $V_{q}=20 \mathrm{MeV}(T=0)$ and $V_{I}(0, T)=16 \mathrm{MeV}(T \neq 0 ; l e f t)$, and $V_{q}=8 \mathrm{MeV}(T=0)$ and $V_{I}(0, T)=6 \mathrm{MeV}$

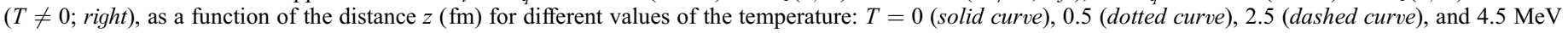
(long-dashed curve). 
$L_{\|}, \theta_{\mathrm{ms}}^{2}=\left(\epsilon_{s} / \epsilon\right)^{2}\left(L_{\|} / X_{0}\right)$, where $\epsilon_{s}=m_{e}(4 \pi / \alpha)^{1 / 2}$ and $X_{0}=$ $\left[4 n \alpha r_{e}^{2} \ln (184)\right]^{-1}$ is the radiation length, is greater than or equal to $\theta_{\gamma}^{2}\left(\theta_{\mathrm{ms}}^{2} \geq \theta_{\gamma}^{2}\right.$; Klein 1999; Hansen et al. 2003; Anthony et al. 1995). Hence the radiation emission differential cross section for the production of a photon is suppressed when

$$
\omega<\omega_{\mathrm{LPM}}=\frac{\epsilon^{2}}{\epsilon_{\mathrm{LPM}}},
$$

where

$$
\epsilon_{\mathrm{LPM}}=\frac{m_{e}^{2} X_{0} \alpha}{8 \pi}
$$

For the electrosphere of the quark stars, in the calculation of the LPM critical frequency $\omega_{\text {LPM }}$ the effect of the external electric field also has to be included. By assuming that the temperature of the star $T \ll p_{\mathrm{F}}=\mu_{e}$, where $p_{\mathrm{F}}$ is the Fermi momentum, the Fermi distribution factors force all the electrons to have $\langle\epsilon\rangle \approx$ $\mu_{e} \approx V$. Therefore, the critical ( $z$-dependent) LPM frequency for the bremsstrahlung photon emission in the electron layer at the surface of the quark star is given by

$$
\begin{aligned}
\omega_{\mathrm{LPM}}(z, T) & \approx \frac{\mu_{e}^{2}(z, T)}{\epsilon_{\mathrm{LPM}} \zeta^{4 / 3}} \approx \frac{V^{2}(z, T)}{\epsilon_{\mathrm{LPM}} \zeta^{4 / 3}} \\
& =\frac{32 r_{e}^{2} \ln 184}{3 \pi m_{e}^{2} \zeta^{4 / 3}} V^{3}(z, T)\left[V^{2}(z, T)+\pi^{2} T^{2}\right],
\end{aligned}
$$

where the parameter $\zeta=e E \epsilon / m_{e}^{3}=\gamma E / E_{\mathrm{cr}}$ takes into account the modifications of the LPM critical frequency due to the presence of the external electric field (Baryshevskii \& Tikhomirov 1986). The LPM frequency for free electrons (in the absence of an external field) is obtained by taking the limit $\zeta \rightarrow 1$ in equation (32). The case $\zeta<1$ corresponds to the classical limit, when the effects of the electric field can be neglected. Therefore, the effect of the electric field on the electromagnetic emission in the electrosphere of the quark stars plays an important role only for values of the electric potential satisfying the condition

$$
V^{2} \sqrt{V^{2}+\pi^{2} T^{2}}>\frac{m_{e}^{3} \sqrt{3 \pi / 2 \alpha}}{e} .
$$

Alternatively, the above condition can be formulated as a general condition for the electric field in the electrosphere, $E>$ $E_{\text {cr }} / \gamma$. In the limit of small temperatures $T \rightarrow 0$, we have $V>$ $V_{\text {crit }}$, where

$$
V_{\text {crit }}=\frac{m_{e}(3 \pi / 2 \alpha)^{1 / 6}}{e^{1 / 3}}=3.40 \mathrm{MeV} .
$$

For $V<V_{\text {crit }}$ the influence of the electric field of the electrosphere on the radiation processes is negligible.

As one can see from equation (32), all the intrinsic emission properties of the electrosphere of the quark stars are determined by a single parameter, the surface electric potential $V(z, T)$. The LPM frequency also rapidly increases with the temperature.

We estimate first the numerical value of the critical frequency for the electrons in the dense layer near the quark star's surface, for $z \approx 0$. In this limit $V(z, T) \rightarrow V_{I}(0, T)$, and by neglecting the effect of the external electric field the LPM frequency is given by

$$
\omega_{\mathrm{LPM}}^{E=0}(0, T)=\left(\frac{32 r_{e}^{2} \ln 184}{3 \pi m_{e}^{2}}\right) V_{I}^{3}(0, T)\left[V_{I}^{2}(0, T)+\pi^{2} T^{2}\right] .
$$

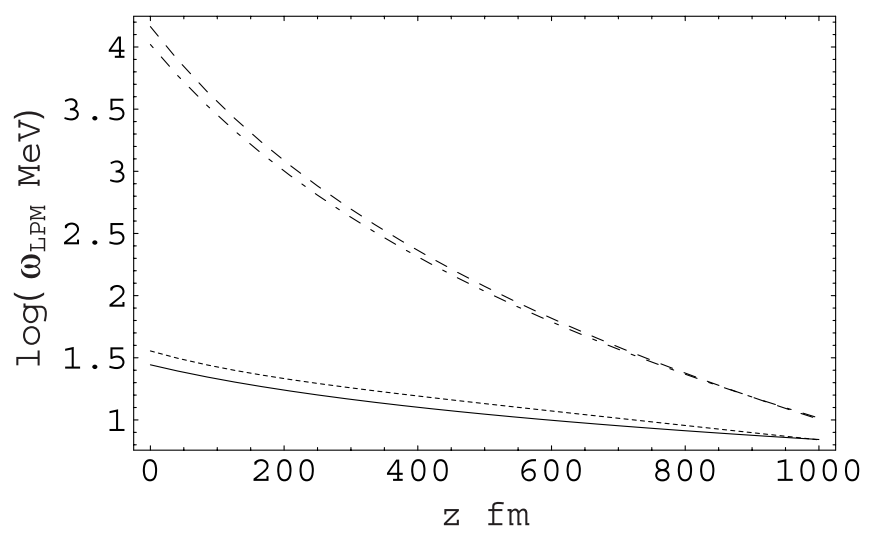

FIG. 3.- LPM frequency $\omega_{\text {LPM }}$ (in a logarithmic scale) of the radiation emitted in the electrosphere of the quark stars in the presence and in the absence of the electric field, for different values of the temperature: $T=0$ and $E \neq 0$ (solid curve), $T=1.5 \mathrm{MeV}$ and $E \neq 0$ (short-dashed curve), $T=0$ and $E=0$ (dash-dotted curve), and $T=1.5 \mathrm{MeV}$ and $E=0$ (long-dashed curve). For the electric potential of the quark star surface we have considered the values $V_{q}=20 \mathrm{MeV}(T=0)$ and $V_{I}(0, T)=16 \mathrm{MeV}(T \neq 0)$.

By adopting a value of the electric potential at the star's surface of the order of $V_{I}(0, T)=16 \mathrm{MeV}$, we obtain $\omega_{\mathrm{LPM}}^{E=0}(0,0) \approx$ $14.5 \mathrm{GeV}$ for $T=0, \omega_{\mathrm{LPM}}^{E=0}(0,1) \approx 15 \mathrm{GeV}$ for $T=1 \mathrm{MeV}$, and $\omega_{\mathrm{LPM}}^{E=0}(0,10) \approx 70 \mathrm{GeV}$ for $T=10 \mathrm{MeV}$. However, by adopting for the electric potential at the star's surface a value of the order of $V_{I}(0, T)=1 \mathrm{MeV}$, the value of the LPM frequency is only $\omega_{\mathrm{LPM}}^{E=0}(0,0) \approx 0.013 \mathrm{MeV}$ for $T=0, \omega_{\mathrm{LPM}}^{E=0}(0,1) \approx 0.15 \mathrm{MeV}$, and $\omega_{\mathrm{LPM}}^{E=0}(0,10) \approx 13.6 \mathrm{MeV}$ for $T=10 \mathrm{MeV}$.

The presence of the electric field in the electrosphere of the quark stars strongly suppresses the LPM effect. For $V_{I}=16 \mathrm{MeV}$ and for $T=0$ the value of the electric field is $E=10 \mathrm{MeV}^{2}$, and $\zeta^{4 / 3}=488$. The corresponding value of the LPM frequency $\omega_{\mathrm{LPM}^{E}}^{E \neq 0}(0,0) \approx 29.61 \mathrm{MeV}$. For $T=1 \mathrm{MeV}, \zeta^{4 / 3}=$ 500.5 and $\omega_{\mathrm{LPM}}^{E \neq 0}(0,1) \approx 30 \mathrm{MeV}$, while for $T=10 \mathrm{MeV}$, $\omega_{\mathrm{LPM}}^{E \neq 0}(0,10) \approx 29.61 \mathrm{MeV}, \zeta^{4 / 3}=1400$, and $\omega_{\mathrm{LPM}}^{E \neq 0}(0,1) \approx$ $50.15 \mathrm{MeV}$. For $V_{I}=1 \mathrm{MeV}$, since $V_{I}<V_{\text {crit }}$, the values of the LPM frequency are the same as in the case of the free electrons, $\left|\omega_{\mathrm{LPM}}^{E \neq 0}(0, T)\right|_{V_{I}=1 \mathrm{MeV}} \approx\left|\omega_{\mathrm{LPM}}^{E=0}(0, T)\right|_{V_{I}=1 \mathrm{MeV}}$.

Emission of electromagnetic radiation with a frequency smaller than $\omega_{\text {LPM }}$ from the electron layer of the quark star surface is suppressed because of the effect of multiple scattering. As expected, the LPM frequency decreases with the density of the electrons at the star's surface, and for $z \geq 500 \mathrm{fm}$ the effect of the multiple scattering can be neglected. The variation of the LPM frequency as a function of $z$ in the presence and in the absence of an electric field is presented, for two different values of the temperature, in Figure 3.

The mean value of the LPM frequency, $\left\langle\omega_{\text {LPM }}\right\rangle$, can be easily obtained in the low-temperature limit, $T \rightarrow 0$. In this case, by neglecting the effect of the external electric field and by taking into account that the effect of multiple scattering has a significant effect on the electron radiation only for a distance of the order of $z_{\text {crit, }}$, we obtain

$$
\left\langle\omega_{\mathrm{LPM}}\right\rangle_{E=0}=\frac{27}{32} \sqrt{\frac{3}{2 \pi \alpha}} \frac{r_{e}^{2} \ln 184}{m_{e}^{2} z_{\text {crit }}} V_{q}^{4}\left[1-\left(1+\frac{\sqrt{6 \alpha} z_{\text {crit }} V_{q}}{4 \sqrt{\pi}}\right)^{-4}\right] .
$$

The value of $z_{\text {crit }}$ can be obtained as a solution of the algebraic equation $z_{\text {crit }} n\left(z_{\text {crit }}, T\right)=1 / 16 \pi r_{e}^{2} Z^{2} \ln \left(184 Z^{-1 / 3}\right)$. The mean frequency of the emitted radiation essentially depends on 


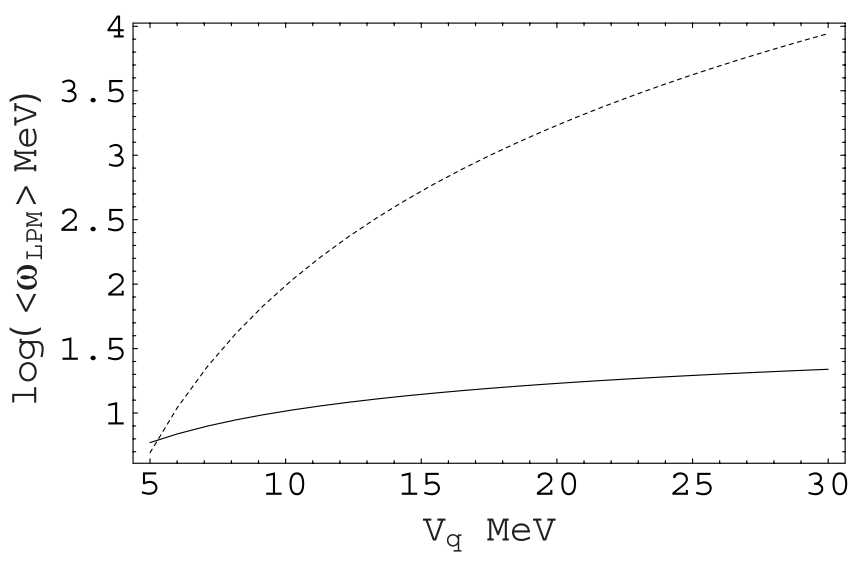

FIG. 4.- Mean LPM frequency $\left\langle\omega_{\mathrm{LPM}}\right\rangle$ (in a logarithmic scale) of the radiation emitted in the electrosphere of cold quark stars $(T<40 \mathrm{MeV})$ as a function of the quark star surface electrostatic potential $V_{q}$ in the presence of a constant external electric field, $E \neq 0$ (solid curve), and with the effects of the external field neglected, $E=0$ (dotted curve).

the value of the electric potential at the quark star surface $V_{q}$ and on the value of the electric field. The mean value of the electron energy in the dense layer of the electrosphere, $\langle\epsilon\rangle=$ $\left(1 / z_{\text {crit }}\right) \int_{0}^{z_{\text {crit }}} V(z) d z$, can be represented as

$$
\langle\epsilon\rangle=\sqrt{\frac{3 \pi}{2 \alpha}} \frac{1}{z_{\text {crit }}} \ln \left(1+\frac{\sqrt{6 \alpha} z_{\text {crit }} V_{q}}{4 \sqrt{\pi}}\right), \quad T=0,
$$

$\langle\epsilon\rangle=\sqrt{\frac{3 \pi}{2 \alpha}} \frac{1}{z_{\text {crit }}} \ln \frac{\left(e^{2 \sqrt{\alpha \pi / 3} T z_{0}}+1\right)\left(e^{2 \sqrt{\alpha \pi / 3} T\left(z_{0}+z_{\text {crit }}\right)}-1\right)}{\left(e^{2 \sqrt{\alpha \pi / 3} T z_{0}}-1\right)\left(e^{2 \sqrt{\alpha \pi / 3} T\left(z_{0}+z_{\text {crit }}\right)}+1\right)}$,

$$
\text { for } T \neq 0 \text {. }
$$

For the mean value of the electric field in the region $0 \leq z \leq z_{\text {crit }}$ we obtain

$$
\langle E\rangle=\frac{3 V_{q}}{4 z_{\text {crit }}}\left[1-\left(1+\frac{\sqrt{6 \alpha} z_{\text {crit }} V_{q}}{4 \sqrt{\pi}}\right)^{-1}\right], \quad T=0
$$

The mean value of the parameter $\zeta=\gamma E / E_{\mathrm{cr}}=e E \epsilon / m_{e}^{3}=$ $(2 \alpha / 3 \pi)^{1 / 2} \mathrm{eV}^{3} / \mathrm{m}_{e}^{3}$, describing the effect of the electric field on

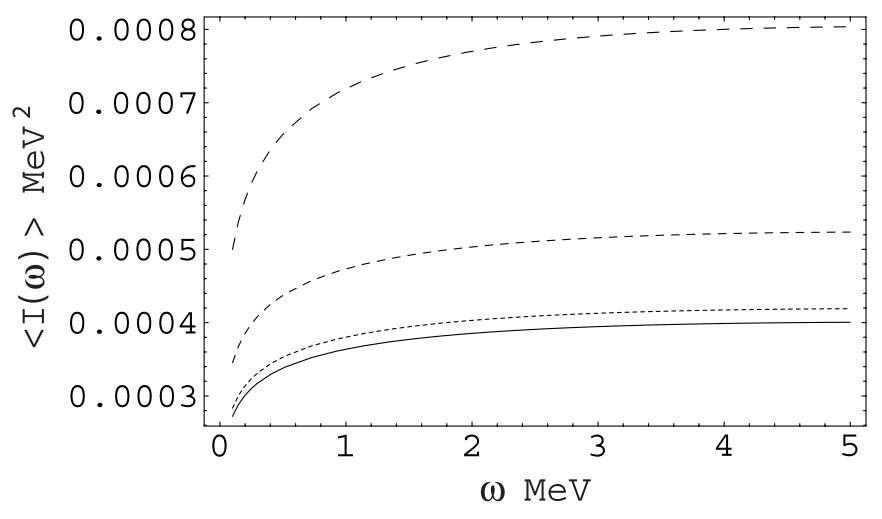

FIG. 6.-Mean intensity $\langle I(\omega)\rangle$ of the radiation emitted by an electron moving in the electric field of the quark star and undergoing multiple scattering as a function of the frequency $\omega$ of the radiation for different values of the temperature: $T=0$ (solid curve), 1.5 (dotted curve), 2.5 (dashed curve), and $4.5 \mathrm{MeV}$ (long-dashed curve). For the value of the quark surface electric potential we have chosen $V_{q}=20 \mathrm{MeV}(T=0)$ and $V_{I}(0, T)=16 \mathrm{MeV}(T \neq 0)$, while $\sigma_{x}=0.1 \mathrm{MeV}$ and $r=2$. For the energy of the electron and the electric field in the electrosphere we have adopted the mean values corresponding to a given temperature.

the radiation processes in the electrosphere of the quark stars, is given by

$$
\begin{gathered}
\langle\zeta\rangle=\frac{27 \pi}{64 \alpha m_{e} E_{\mathrm{cr}}} \sqrt{\frac{3 \pi}{2 \alpha}} \frac{V_{q}^{2}}{z_{\text {crit }}}\left[1-\left(1+\frac{\sqrt{6 \alpha} z_{\text {crit }} V_{q}}{4 \sqrt{\pi}}\right)^{-2}\right] \\
\text { for } T=0 .
\end{gathered}
$$

By including the effect of the external electric field, the mean value of the LPM frequency can be expressed as

$$
\begin{gathered}
\left\langle\omega_{\mathrm{LPM}}\right\rangle_{E \neq 0}=\frac{32(3 \pi)^{1 / 6} r_{e}^{2} E_{\mathrm{cr}} \ln 184}{(2 \alpha)^{7 / 6} e^{1 / 3} z_{\text {crit }}} \ln \left(1+\frac{\sqrt{6 \alpha} z_{\text {crit }} V_{q}}{4 \sqrt{\pi}}\right), \\
\text { for } T=0 .
\end{gathered}
$$

In the absence of the electric field, the dependence of the LPM critical frequency on the surface potential of the quark star is $\left\langle\omega_{\text {LPM }}\right\rangle \sim V_{q}^{4}$, while in the presence of the electric field, we have a logarithmic dependence on the quark star potential. The dependence of the mean LPM critical frequency $\left\langle\omega_{\text {LPM }}\right\rangle$ on the quark star surface potential $V_{q}$, in the low-temperature limit and
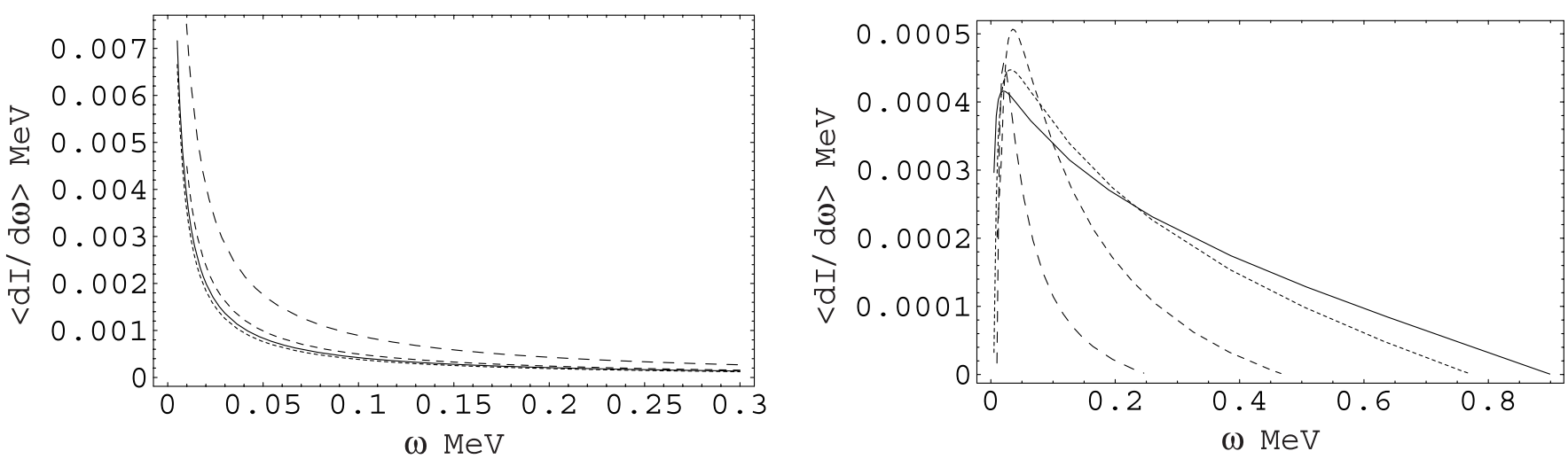

FIG. 5.-Frequency distribution of the mean intensity $\langle d I / d \omega\rangle$ of the radiation emitted by an electron moving in the exterior electric field of the quark star and

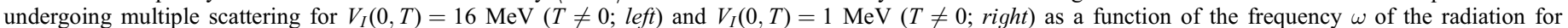

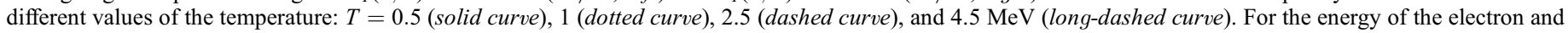
the electric field in the electrosphere we have adopted the mean values corresponding to a given temperature, while $\sigma_{x}=0.1 \mathrm{MeV}$ and $r=2$. 

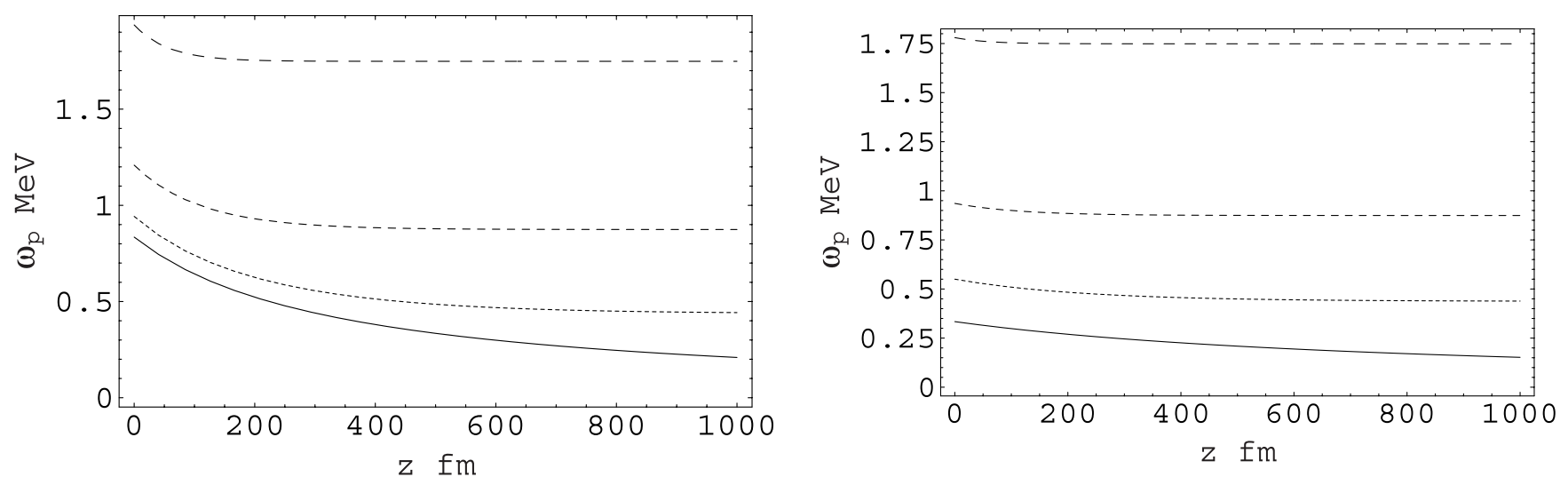

FIG. 7.-Plasma frequency $\omega_{p}$ of the electrosphere of the quark stars as a function of the distance $z$ for $V_{q}=20 \mathrm{MeV}(T=0)$ and $V_{I}(0, T)=16 \mathrm{MeV}(T \neq 0$; left), and $V_{q}=8 \mathrm{MeV}(T=0)$ and $V_{I}(0, T)=6 \mathrm{MeV}(T \neq 0$; right), for different values of the temperature: $T=0$ (solid curve), 2.5 (dotted curve), 5 (dashed curve), and $10 \mathrm{MeV}$ (long-dashed curve).

with and without the presence of an external electric field, is presented in Figure 4.

In order to calculate the spectral distribution of the intensity of the electromagnetic radiation emitted by an electron in the dense layer of the electrosphere of a quark star, taking into account the effect of the multiple collisions and of the electric field, we use equations (17)-(21). The variation of the probability of emission of a photon by an electron moving in the electrosphere of the quark star is presented, for two sets of values of the quark star surface electric potential, in Figure 5. The mean value of the total intensity of the radiation $\langle I(\omega)\rangle$ is presented in Figure 6.

An important parameter describing the radiation properties of an electron gas is the plasma frequency $\omega_{p}$, which is defined as $\omega_{p}=\left[4 \pi e n_{e}(z, T) / \mu_{e}(z, T)\right]^{1 / 2}$ and which is related to the medium permittivity $\varepsilon(\omega)$ by $\varepsilon(\omega) \approx 1-\omega_{p}^{2} / \omega^{2}, \omega \gg \omega_{p}$ (Jackson 1999). The polarization of the electrosphere of the quark stars also strongly influences the radiation processes, and photons with frequency $\omega<\omega_{p}$ cannot escape from the electrosphere. The variation of the plasma frequency of the quark star electrosphere is presented as a function of distance in Figure 7.

The variation of the emissivity of the electrosphere of the quark star, fully taking into account the effect of the electric field on the suppression of the radiation due to the multiple scattering effects, is presented in Figure 8. The total energy flux of the electrosphere is obtained as $F_{\gamma}^{(e e)}=(1 / \pi) \int_{0}^{\infty} \varepsilon_{\gamma}^{(e e)} d z$. The variation of the energy flux as a function of the temperature of the quark star surface is presented, for different values of the surface electric potential $V_{I}$, in Figure 9.

\section{DISCUSSION AND FINAL REMARKS}

Since the proposal of strange quark stars, much effort has been devoted to finding major observational properties that distinguish strange stars from neutron stars. One of the important difference between them is the stellar radius. Strange stars could have a radius significantly smaller than that of neutron stars. The observed thermal X-ray properties of compact objects may be a possible probe for determining the stellar radius. In the present paper we have shown that the high-density degenerate electron layer at the surface of a bare strange star could play an important role in the electromagnetic radiation emission from the star. The bremsstrahlung emissivity of the electrosphere is generally a function of the electron density, the temperature, and the electrostatic potential at the surface of the star. The bremsstrahlung emission spectrum is very different from the blackbody spectrum. For hot strange stars, the energy emission from the electrosphere could exceed the intensity of the blackbody radiation. Even in the low-temperature limit the photon emission from the electron layer exceeds the bremsstrahlung emissivity of the quark-gluon plasma. This makes the process of electronelectron bremsstrahlung of considerable importance for establishing the correct observational signatures of quark stars. Since the photon emission from the strange star's surface is dominated by the bremsstrahlung emission from the electron layer, it is
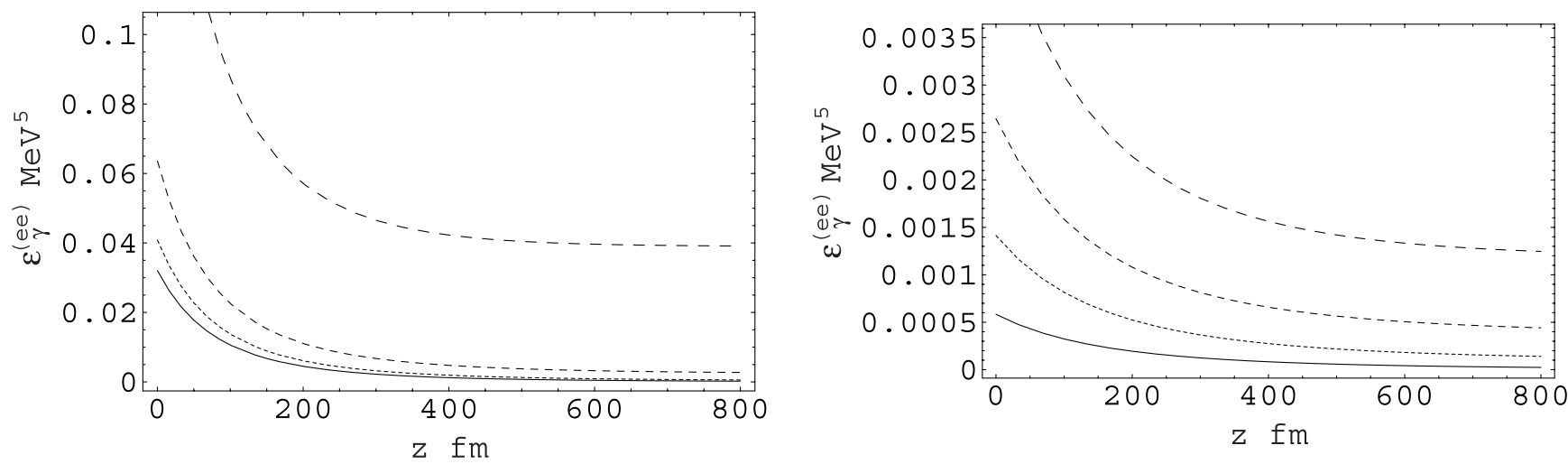

FIG. 8.-Emissivity $\varepsilon_{\gamma}^{(e e)}(z, T)$ of the electrosphere of the quark stars as a function of the radial distance $z$ for $V_{I}=16 \mathrm{MeV}(T \neq 0 ; l e f t)$ and $V_{I}(0, T)=8 \mathrm{MeV}$ $(T \neq 0 ;$ right), for different values of the temperature: $T=0.5$ (solid curve), 1.5 (dotted curve), 2.5 (dashed curve), and $4.5 \mathrm{MeV}$ (long-dashed curve). For the energy of the electron and the electric field in the electrosphere we have adopted the mean values corresponding to a given temperature, while $\sigma_{x}=0.1 \mathrm{MeV}$ and $r=2$. 


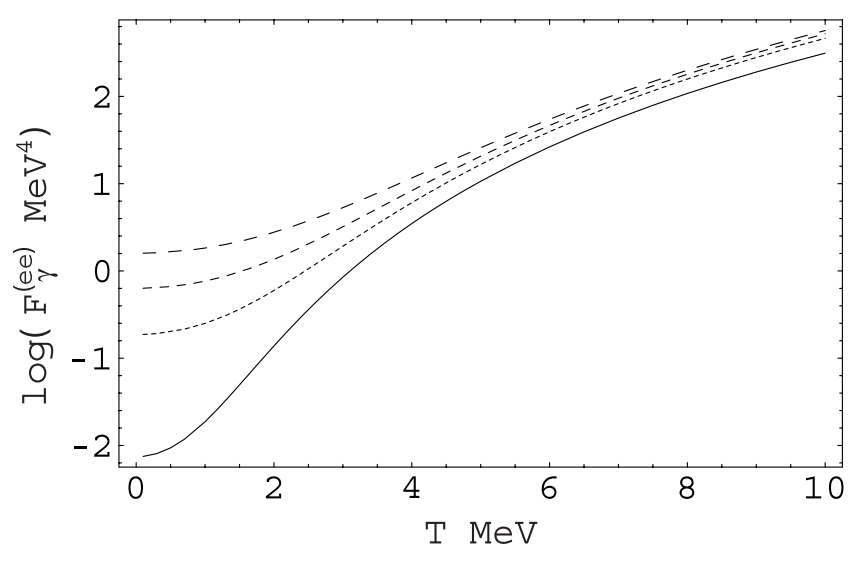

FIG. 9.- Variation, as a function of the temperature $T$, of the energy flux $F_{\gamma}^{(e e)}$ emitted by the electrosphere of a quark star (in a logarithmic scale), for different values of the quark star surface electric potential $V_{I}: V_{I}=8$ (solid curve), 12 (dotted curve), 16 (dashed curve), and $20 \mathrm{MeV}$ (long-dashed curve). For the energy of the electron and the electric field in the electrosphere we have adopted the mean values corresponding to a given temperature, while $\sigma_{x}=0.1 \mathrm{MeV}$ and $r=2$.

necessary to include this effect in determining the radius of the star.

One of the important features of the bremsstrahlung radiation from a single interaction is the independence of $d I / d \omega$ on $\omega$ (Landau \& Lifshitz 1975). However, as one can see from equation (14), in the case of the strongly suppressed radiation of a particle in a dense medium we have $d I / d \omega \propto \omega^{1 / 2}$. Therefore, because of the multiple scattering effects, the spectrum of the radiation of the dense electrosphere differs in a qualitative way from the standard bremsstrahlung. On the other hand, the electric field of the electrosphere decreases the LPM critical frequency, thus leading to an increase of the electromagnetic energy flux from the bare strange star surface.

The results for the bremsstrahlung radiation of the electrosphere obtained in the present paper have been obtained under two main assumptions. First, we have considered the equations for the radiation emission process derived for the case of a constant electric field. The electric field in the electrosphere is not constant, and in order to take into account the variations of $E$ and of the energies of the electrons, we have adopted the mean values of these quantities. This approximation is, of course, not generally valid for all layers of the electrosphere, but we still expect that it can provide a general description of the radiation pattern of the electron layer. Second, in describing the LPM effect, we have adopted for the electrons the formulas and equations describing multiple Coulomb scattering in amorphous media, where the main contribution to scattering comes from fixed centers, such as the nuclei or the crystalline lattice. To apply this well-established formalism to the case of the electrosphere of the strange stars, we have assumed that the multiple scattering processes between electrons can be described by taking $Z=1$ in the equations describing multiple collisions and radiation suppression in amorphous media. In other words, we approximate the multiple collision and scattering processes between electrons in the dense electrosphere as scattering of an electron on a fixed center. The differences between these physical processes could affect the numerical estimations for the suppression factor, critical LPM frequency, and radiation spectrum obtained in the present paper. However, we expect that the results obtained in the present paper could give a good qualitative description of the radiation emission processes from the electrosphere.

The radiation properties of the electrosphere and the LPM suppression effect essentially depend on the numerical value of the electrostatic potential at the quark star surface $V_{I}(0, T)$, which at low temperatures is related to the quark electric potential $V_{q}$ in the bulk. It is now commonly accepted that because of the attractive interaction between quarks in some specific channels, the ground state of the strange quark matter is a color superconductor, which at asymptotic densities is in the CFL phase, in which quarks of all three flavors and three colors are paired in a single condensate. The CFL phase in the bulk consists of equal numbers of $u, d$, and $s$ quarks and is electrically neutral in the absence of any electrons, that is, in this state $V_{q}=0$ (Alford et al. 1999; Rajagopal \& Wilczek 2001). Therefore, for strange stars in the CFL phase there could be no surface electron layer, as suggested by Lugones \& Horvath (2003).

On the other hand, the density of quark states near the surface of a strange star differs from the density of quark states in the bulk (Madsen 2000b, 2001). This results in a sharp increase of quark charge density at the star's surface. Because of the surface depletion of the $s$ quarks, a thin charged layer forms at the surface of the star. Because of this surface effect the electric field and the density of electrons could increase by an order of magnitude (Usov 2004; Usov et al. 2005). The inclusion of the surface or other effects in the electrosphere models would also require the reconsideration of the radiation emission processes, taking into account their influence on the radiation suppression mechanisms and on the critical LPM frequency.

This work is supported by a RGC grant of the Hong Kong Government. We are grateful to the anonymous referee for comments that significantly improved the manuscript.
Akhiezer, A. I., \& Shul'ga, N. F. 1996, High-Energy Electrodynamics in Matter (New York: Gordon \& Breach)

Alcock, C., Farhi, E., \& Olinto, A. 1986, ApJ, 310, 261

Alford, M., Rajagopal, K., \& Wilczek, F. 1998, Phys. Lett. B, 422, 247 1999, Nucl. Phys. B, 537, 443

Anthony, P. L., et al. 1995, Phys. Rev. Lett., 75, 1949

Bailin, D., \& Love, A. 1984, Phys. Rep., 107, 325

Baryshevskii, V. G., \& Tikhomirov, V. V. 1986, Soviet Phys.-JETP, 63, 1116

Berestetskii, V. B., Lifshitz, E. M., \& Pitaevskii, L. P. 1982, Quantum Electrodynamics (2nd ed; Oxford: Pergamon)

Blaschke, D., Klahn, T., \& Voskresensky, D. N. 2000, ApJ, 533, 406

Bodmer, A. 1971, Phys. Rev. D, 4, 1601

Cheng, K. S., \& Dai, Z. G. 1996, Phys. Rev. Lett., 77, 1210 1998, Phys. Rev. Lett., 80, 18

Cheng, K. S., Dai, Z. G., \& Lu, T. 1998a, Int. J. Mod. Phys. D, 7, 139

Cheng, K. S., Dai, Z. G., Wei, D. M., \& Lu, T. 1998b, Science, 280, 407

\section{REFERENCES}

Cheng, K. S., \& Harko, T. 2000, Phys. Rev. D, 62, 083001 2003, ApJ, 596, 451

Chiu, H.-Y. 1968, Stellar Physics (Waltham: Blaisdell)

Chmaj, T., Haensel, P., \& Slominski, W. 1991, Nucl. Phys. B Proc. Suppl., 24, 40

Dai, Z. G., Peng, Q. H., \& Lu, T. 1995, ApJ, 440, 815

Dey, M., Bombacci, I., Dey, J., Ray, S., \& Samanta, B. C. 1998, Phys. Lett. B, 438,123

Gondek-Rosinska, D., Bulik, T., Zdunik, L., Gourgoulhon, E., Ray, S., Dey, J., \& Dey, M. 2000, A\&A, 363, 1005

Gourgoulhon, E., Haensel, P., Livine, R., Paluch, E., Bonazzola, S., \& Marck, J.-A. 1999, A\&A, 349, 851

Ha, T. B. 1997, Phys. Lett. B, 396, 269

Haensel, P., Zdunik, J. L., \& Schaeffer, R. 1986, A\&A, 160, 121

Hansen, H. D., Uggerhj, U. I., Biino, C., Ballestrero, S., Mangiarotti, A., Sona, P., Ketel, T. J., \& Vilakazi, Z. Z. 2003, Phys. Rev. Lett., 91, 014801 
Harko, T., \& Cheng, K. S. 2002a, A\&A, 385, 947 2002b, MNRAS, 335, 99

Harko, T., Cheng, K. S., \& Tang, P. S. 2004, ApJ, 608, 945

Itoh, N. 1970, Prog. Theor. Phys., 44, 291

Jackson, J. D. 1999, Classical Electrodynamics (3rd ed.; New York: Wiley)

Jaikumar, P., Gale, C., Page, D., \& Prakash, M. 2004, Phys. Rev. D, 70, 023004 2005, Int. J. Mod. Phys. A, in press (astro-ph/0407091)

Kettner, Ch., Weber, F., Weigel, M. K., \& Glendenning, N. K. 1995, Phys. Rev. D, 51,1440

Klein, S. 1999, Rev. Mod. Phys., 71, 1501

Landau, L. D., \& Lifshitz, E. M. 1975, The Classical Theory of Fields (4th ed.; Oxford: Pergamon)

Landau, L. D., \& Pomeranchuk, I. J. 1953, Dokl. Akad. Nauk SSSR, 92, 735

Lugones, G., \& Horvath, J. E. 2003, A\&A, 403, 173
Madsen, J. 2000a, Phys. Rev. Lett., 85, 10 2000b, Phys. Rev. Lett., 85, 4687 2001, Phys. Rev. Lett., 87, 172003

Migdal, A. B. 1956, Phys. Rev., 103, 1811

Page, D., \& Usov, V. V. 2002, Phys. Rev. Lett., 89, 131101

Rajagopal, K., \& Wilczek, F. 2001, Phys. Rev. Lett., 86, 3492

Terazawa, H. 1979, INS Rep. 336 (Tokyo: Univ. Tokyo, INS)

Usov, V. V. 1998a, Phys. Rev. Lett., 80, 230 1998b, Phys. Rev. Lett., 81, 4775 2001, ApJ, 550, L179 2004, Phys. Rev. D, 70, 067301

Usov, V. V., Harko, T., \& Cheng, K. S. 2005, ApJ, 620, 915

Witten, E. 1984, Phys. Rev. D, 30, 272

Zdunik, J. L. 2000, A\&A, 359, 311 